\title{
Article \\ Comparative Study of Anti-Inflammatory Effect on DSS-Induced Ulcerative Colitis Between Novel Glycyrrhiza Variety and Official Compendia
}

Sa-Haeng Kang ${ }^{1}$, Young-Jae Song ${ }^{1}$, Yong-Deok Jeon ${ }^{2}$, Dong-Keun Kim ${ }^{1}$, Jeong-Hyang Park ${ }^{1}$, Ju-Ryoun Soh ${ }^{1}$, Jong-Hyun Lee ${ }^{3}$, Christopher Kitalong ${ }^{4}$, Wonnam Kim ${ }^{5}$, Hyo-Jin An ${ }^{6}$, Jae-Ki Chang ${ }^{7}$, Jeonghoon Lee ${ }^{7}$ * and Jong-Sik Jin $1, *$ (D)

check for updates

Citation: Kang, S.-H.; Song, Y.-J.; Jeon, Y.-D.; Kim, D.-K.; Park, J.-H.; Soh, J.-R.; Lee, J.-H.; Kitalong, C.; Kim, W.; An, H.-J.; et al. Comparative Study of Anti-Inflammatory Effect on DSS-Induced Ulcerative Colitis Between Novel Glycyrrhiza Variety and Official Compendia. Appl. Sci. 2021, 11, 1545. https://doi.org/ 10.3390/app11041545

Academic Editor: Natália Martins

Received: 11 January 2021

Accepted: 27 January 2021

Published: 8 February 202

Publisher's Note: MDPI stays neutral with regard to jurisdictional claims in published maps and institutional affiliations.

Copyright: (c) 2021 by the authors Licensee MDPI, Basel, Switzerland. This article is an open access article distributed under the terms and conditions of the Creative Commons Attribution (CC BY) license (https:/ / creativecommons.org/licenses/by/ $4.0 /)$
1 Department of Oriental Medicine Resources, Jeonbuk National University, 79 Gobong-ro, Iksan, Jeollabuk-do 54596, Korea; kangsh@jbnu.ac.kr (S.-H.K.); dudwoid@naver.com (Y.-J.S.); kdk5796@daum.net (D.-K.K.); rollipope@hanmail.net (J.-H.P.); jrsoh@nate.com (J.-R.S.)

2 Department of Korean Pharmacy, College of Pharmacy, Woosuk University, 443 Samrye-ro, Samrye-eup, Wanju-gun, Jeollabuk-do 55338, Korea; ydjeon1211jh@woosuk.ac.kr

3 Department of Pharmacy, College of Pharmacy, Dongduk Women's University, 60 Hwarang-ro 13-gil, Seongbuk-gu, Seoul 02748, Korea; naturalmed@dongduk.ac.kr

4 Pacific Academic Institute for Research, Palau Community College-Cooperative Research Extension, 9 Main Street, Koror 96940, Palau; ckitalon@gmail.com

5 Division of Pharmacology, College of Korean Medicine, Semyung University, 65 Semyung-ro, Jecheon, Chungbuk 27136, Korea; wonnam_kim@semyung.ac.kr

6 Department of Pharmacology, College of Korean Medicine, Sangji University, 83 Sangjidae-gil, Wonju-si, Gangwon-do 26339, Korea; sangjipharm@gmail.com

7 Herbal Crop Research Division, NIHHS, RDA, Eumseong, Chungbuk 27709, Korea; changjk@korea.kr

* Correspondence: artemisia@korea.kr (J.L.); jongsik.jin@jbnu.ac.kr (J.-S.J.)

Abstract: Glycyrrhizae radix (GR), a plant commonly referred to as licorice, is used as a medicine and food worldwide. However, the utilization of GR from wild areas has caused desertification and a depletion of natural resources. Environmental restrictions and low productivity have limited plant cultivation. For this reason, an improved Glycyrrhiza variety, Wongam (WG), in cultivation and quality has been developed by Korea Rural Development Administration. To evaluate the equivalence of efficacy, several comparative studies between already-registered species and new cultivars have been conducted. This study evaluated the anti-inflammatory effect of WG extracts in a dextran sulfate sodium (DSS)-induced colitis model, in comparison to that of GR extracts. WG extract significantly improved the clinical signs of DSS-induced ulcerative colitis, including disease activity index, body weight loss, and colon length shortening, which was equivalent to the effect of GR. Furthermore, the fecal microbiota was analyzed by terminal restriction fragment length polymorphism. The composition of the fecal microbiota did not show a specific pattern based on experimental groups; however, a tendency toward an increase in the proportion of Lactobacillales was observed. These findings showed an equivalence of efficacy and the possible utilization of WG as a medicinal resource with already-registered species.

Keywords: improved glycyrrhiza varieties; wongam; ulcerative colitis; inflammation; fecal microbiota

\section{Introduction}

Glycyrrhizae radix (GR) has long been used as a medicinal and edible resource. Many pharmacological effects of GR have been studied and applied to industrial products, such as medicines, foods, and cosmetics [1-3]. As GR has increasingly been utilized, wild GR has been collected in Central Asia, causing serious desertification and the depletion of natural resources. Much of the GR in the market is agricultural; however, environmental restriction and low productivity of GR limit the cultivation. To resolve these problems, the Korea Rural Development Administration developed a novel variety of Glycyrrhiza, a Glycyrrhiza 
glabra $\times$ Glycyrrhiza uralensis hybrid [4] named Wongam (WG) and Sinwongam (SWG). The variety showed stability in glycyrrhizin and liquiritigenin content, suitability for cultivation, and productivity.

To extend the utilization of the improved variety, the safety and equivalence of efficacy between Glycyrrhiza species have been intensively studied. Recent studies have shown anti-inflammatory, antioxidant, and cognitive effects of WG and SWG [5]. These effects were of an equivalent level to those for registered Glycyrrhiza species.

It is well known that inflammatory bowel diseases (IBDs), including Crohn's disease (CD) and ulcerative colitis (UC), are chronic, recurrent inflammatory disorders of the gastrointestinal tract [6,7], characterized by an infiltration of neutrophils into the lesion of the colon accompanied by epithelial cell necrosis and ulceration [8-10]. Although the exact pathogenicity of IBDs is not well known, there is some proof that it involves interaction among the genetic susceptibility, immune system, and the environment, most notably the bacterial flora. Patients with UC show many clinical symptoms, such as abdominal pain, fecal blood, weight loss, and diarrhea [11-14]. To study this, a type of experimental colitis that exhibits morphological changes similar to human UC symptoms can be induced in mice through drinking water containing dextran sulfate sodium (DSS) [15]. This model is efficient and has thus been utilized in screening synthetic compounds for regulating colitis, including UC [16]. Common therapies for UC include sulfasalazine, glucocorticoids, and immunosuppressants but have various systemic side effects [17]. The inflammatory response in UC is caused by the activation of macrophages by bacterial products such as lipopolysaccharides (LPS) [18]. Activated macrophages release pro-inflammatory cytokines such as interleukin-6 (IL-6) and tumor necrosis factor- $\alpha$ (TNF- $\alpha$ ). Patients with UC are known to have high levels of inflammatory cytokines such as IL- 6 and TNF- $\alpha$ [19]. However, there are no therapies available to cure IBDs completely yet. Therefore, we intended to study the anti-inflammatory activity by utilizing medicinal plants without side effects.

As part of the study for the evaluation of equivalent efficacy, the anti-inflammatory activity of the new Glycyrrhiza variety, WG, was investigated using a DSS-induced UC mouse model, and the fecal microbiota was analyzed.

\section{Results}

\subsection{Quantification of Glycyrrhizic Acid and Liquiritigenin in WG and $G R$}

When WG and GR were analyzed by LC/MS, glycyrrhizic acid (GA) and liquiritigenin (LG) were detected in both extracts (Figure 1a). The retention times were 5.9 and $9.5 \mathrm{~min}$, respectively (Figure $1 b$ ). The mass fragmentation patterns of the compounds from the WG and GR were identical for each standard compound. In WG and GR extracts, $11.66 \%$ and $11.38 \%$, respectively, of GA and $0.64 \%$ and $0.77 \%$, respectively, of LG were quantified.

\subsection{Effect of WG and GR Extracts on Clinical Signs of UC Induced by DSS}

To measure the effect of WG and GR extracts on UC, WG and GR extracts were administered to a UC mouse model induced by DSS, and changes in body weight were measured. Mice were administered DSS followed by WG extract at concentrations of 10, 50, and $100 \mathrm{mg} / \mathrm{kg}$ for 12 days. The reference compound was 5-aminosalicylic acid (5-ASA). As shown in Figure 2, the greatest weight loss was observed in the group administered with DSS alone. The DSS group showed a body weight decrease (Figure 2a) at Day 12 of $16.2 \mathrm{~g}$, compared to the control. Both WG and GR $100 \mathrm{mg} / \mathrm{kg}$ (WG100 and GR100) alleviated body weight loss and the disease activity index (DAI) score after DSS administration (Figure 2a,b). The DAI scores were also significantly lower in the WG100 and GR100 groups compared with those of the DSS group. The effect of the WG and GR extracts on DAI in the DSS-induced UC model was similar to that of the 5-ASA-administered group (Figure 2b). 


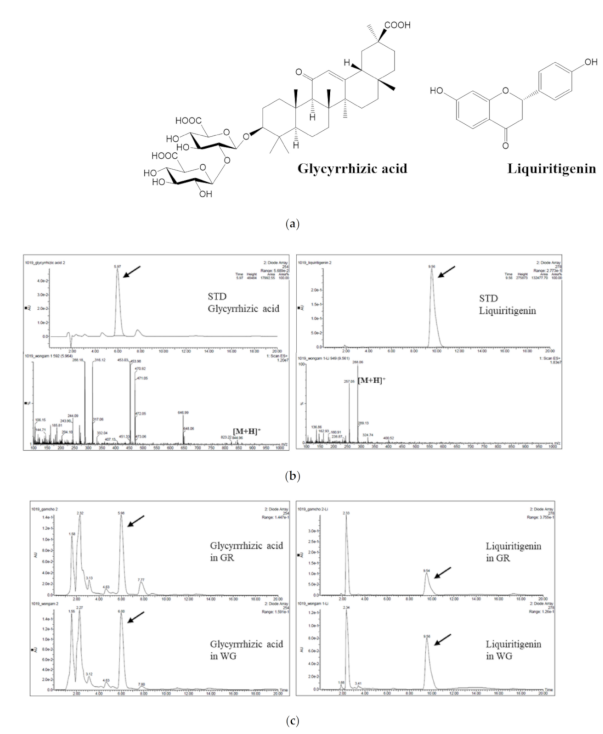

Figure 1. Chemical analysis of glycyrrhizic acid (GA) and liquiritigenin in Wongam (WG) and glycyrrhizae radix (GR): (a) chemical structures of GA and liquiritigenin; (b) HPLC chromatogram and mass fragmentation pattern of GA and liquiritigenin; (c) HPLC chromatogram of WG and GR extracts.

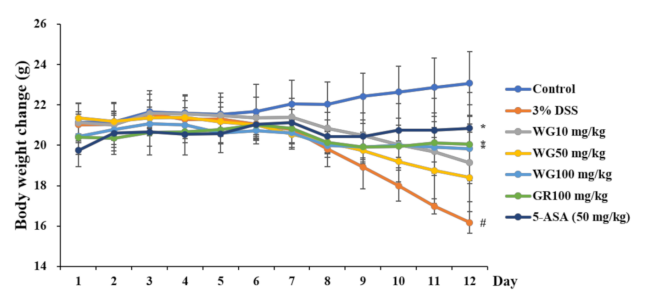

(a)

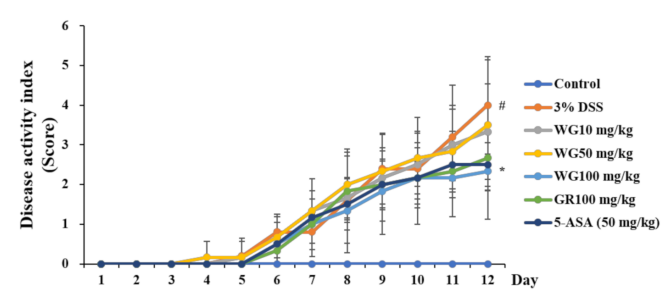

(b)

Figure 2. WG and GR extracts mitigate symptoms of dextran sulfate sodium (DSS)-induced colitis. Mice were divided into groups: control, 3\% DSS-treated, 3\% DSS-treated with WG and GR extracts (10, 50, or $100 \mathrm{mg} / \mathrm{kg}$ for WG and $100 \mathrm{mg} / \mathrm{kg}$ for GR), and 3\% DSS-treated with 5-aminosalicylic acid $(50 \mathrm{mg} / \mathrm{kg}$ ). (a) Weight changes are given in grams (g). (b) Disease activity index (DAI) scores in the seven groups. Data are expressed as mean \pm standard error of the mean (SEM) $(n=6 /$ treatment) (\#: $p<0.05$ vs. control; * $p<0.05$ vs. DSS only).

\subsection{Effects of WG and GR Extracts on Colon Length Shortening in UC Induced by DSS}

In DSS-induced animal models, various physical symptoms will appear, including changes in the length of the large intestine. The length of the colon can be used to indirectly assess the degree of inflammation [20]. In this study, the average colon length of the control was $9.25 \pm 0.38 \mathrm{~cm}$. The colon length in the DSS group was significantly shorter, at $5.96 \pm 0.88 \mathrm{~cm}$, than that in the control group. Colons from the WG100 and GR100 groups were longer $(7.11 \pm 0.61 \mathrm{~cm}$ and $7.02 \pm 0.88 \mathrm{~cm}$, respectively (Figure $3 \mathrm{a}, \mathrm{b})$ than those in the DSS group. 


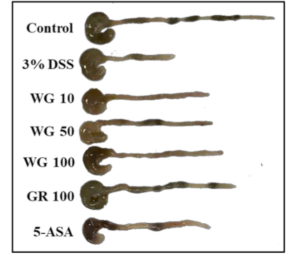

(a)

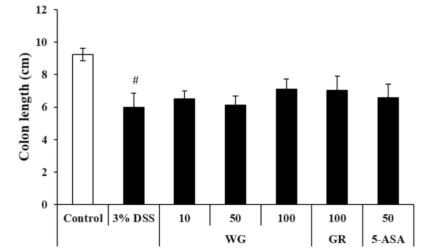

(b)

Figure 3. WG and GR extracts alleviated DSS-induced colon shortening. (a) Macroscopic appearance of colon. (b) Length of colon. Data are expressed as mean $\pm \operatorname{SEM}(n=6 /$ treatment) (\#: $p<0.05$ vs.

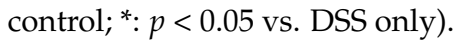

\subsection{Effect of WG and GR Extracts on Serum Levels of Inflammatory Cytokines}

Inflammatory cytokines appear early in the inflammatory response of UC [21]. To determine the effect of WG and GR extracts on the serum levels of IL- 6 and TNF- $\alpha$, ELISAs were conducted. The serum levels of IL-6 were found to be significantly lower in the WG100 group $(14.4 \pm 7.9 \mathrm{pg} / \mathrm{mL})$ than in the DSS group $(87.2 \pm 10.7 \mathrm{pg} / \mathrm{mL})$ (Figure $4 \mathrm{a})$. Furthermore, the serum levels of TNF- $\alpha$ were also significantly lower in the WG100 group $(3842 \pm 188 \mathrm{pg} / \mathrm{mL})$ than those in the DSS group (5214 $\pm 300 \mathrm{pg} / \mathrm{mL})$ (Figure $4 \mathrm{~b})$.

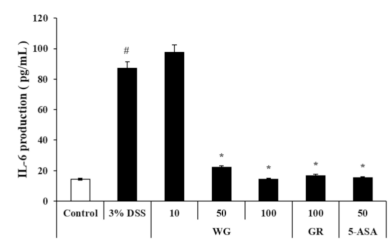

(a)

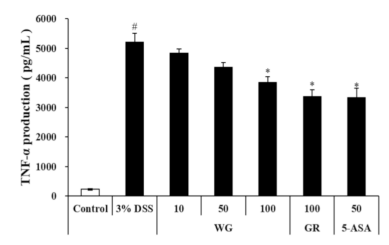

(b)

Figure 4. Effect of WG and GR extracts on interleukin-6 (IL-6) and tumor necrosis factor- $\alpha$ (TNF- $\alpha$ ) generation in DSS-induced colitis. Cytokine assays were used to determine the serum levels of IL-6 and TNF- $\alpha$ : (a) IL-6 levels and (b) TNF- $\alpha$ levels in mouse serum. Data for each experiment are expressed as mean $\pm \operatorname{SEM}(n=6 /$ treatment) (\#: $p<0.05$ vs. control; *: $p<0.05$ vs. DSS only).

\subsection{Effect of WG and GR on PGE 2 Production in Colon Tissue}

$\mathrm{UC}$ patients have shown elevated levels of prostaglandin $\mathrm{E}_{2}\left(\mathrm{PGE}_{2}\right)$ in their intestines [22]. We used the ELISA to determine the effect of WG and GR on the inflammatory mediator $\mathrm{PGE}_{2}$ in mouse colon tissue affected by DSS-induced UC, and $\mathrm{PGE}_{2}$ levels in the DSS group $(1607.52 \pm 128.60 \mathrm{pg} / \mathrm{mL})$ were significantly higher than those of the control group $\left(521.65 \pm 20.86 \mathrm{pg} / \mathrm{mL}\right.$ ). However, $\mathrm{PGE}_{2}$ levels in the WG100 and GR100 groups $(1284.95 \pm 25.69 \mathrm{pg} / \mathrm{mL}$ and $1276.49 \pm 63.85 \mathrm{pg} / \mathrm{mL}$, respectively) were significantly lower than those in the DSS group (Figure 5).

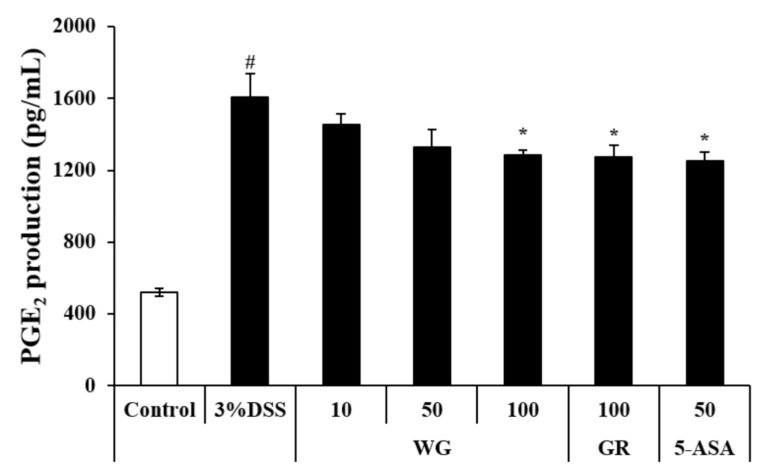

Figure 5. Effect of WG and GR extracts on prostaglandin $\mathrm{E}_{2}\left(\mathrm{PGE}_{2}\right)$ production in colon tissues. $\mathrm{PGE}_{2}$ levels were measured using $\mathrm{PGE}_{2}$ assay kits. Data are expressed as mean $\pm \operatorname{SEM}(n=6 /$ treatment $)$ (\#: $p<0.05$ vs. control; * $p<0.05$ vs. DSS only). 


\subsection{Effects of WG and GR on Epithelial Injury in DSS-Induced Colitis}

Mucosal thickness is considered as a parameter of normal mucosal conditions. DSS causes epithelial injury and penetration of inflammatory cells, including mast cells [23]. The histological damage in colon tissue between the control and DSS-treated groups was evident, so we then examined the effect of treatment with WG and GR extracts. For WG $100 \mathrm{mg} / \mathrm{kg}$, WG attenuated the DSS-induced effects (Figure 6a), while the GR $100 \mathrm{mg} / \mathrm{kg}$ treatment also reduced DSS-mediated damage (Figure $6 \mathrm{~b}$ ). The mucosal thickness measure is regarded as a parameter for assessing mucosal condition. We also found significant increases in mucosal thickness in the DSS group. However, this was significantly decreased in the WG and GR groups based on concentration (Figure 6c).
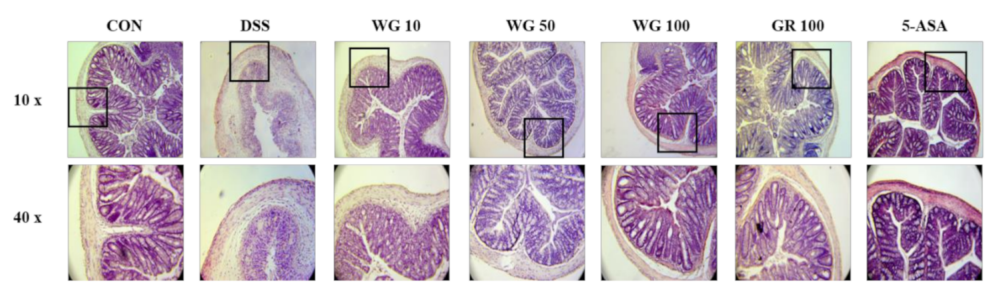

(a)

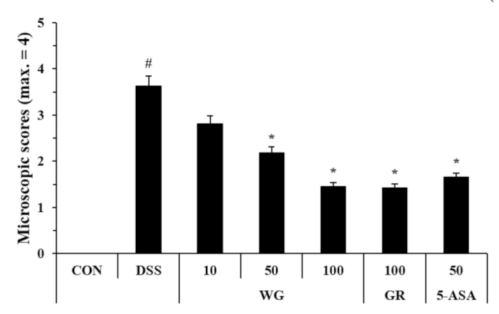

(b)

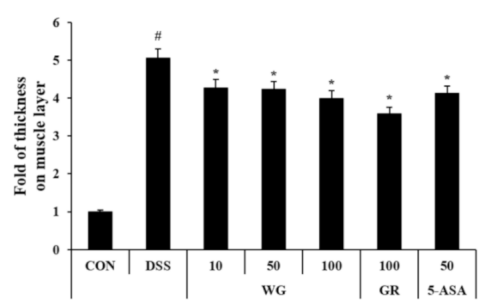

(c)

Figure 6. Effect of WG and GR extracts on epithelial injury in mice with DSS-induced colitis. Over the same period, WG $(10,50$, and $100 \mathrm{mg} / \mathrm{kg}), \mathrm{GR}(100 \mathrm{mg} / \mathrm{kg})$, and the positive control 5-ASA $(50 \mathrm{mg} / \mathrm{kg}$ ) were orally administered once a day. (a) Colon tissue paraffin sections stained with hematoxylin and eosin (H\&E) were observed under a microscope $(10 \times$ and $40 \times)$. (b) Histological score was measured from the colon tissues on Day 12. (c) The thickness of muscle in the colon sections was evaluated using Optiview 3.7 software. The stained sections were observed under a microscope. Values are mean \pm SEM (\#: $p<0.05$ vs. control; * $p<0.05$ vs. DSS only).

\subsection{Effects of WG on Fecal Microbiota in DSS-Induced Colitis Model}

In some fecal samples collected from DSS-treated mice, inhibition of PCR was observed as reported [24]. Therefore, these samples could not be analyzed and merged.

Terminal restriction fragments (T-RFs) were distributed to 29 operational taxonomic units (OTUs), as reported previously $[25,26]$. Five of the OTUs were removed from the list because no T-RFs belonging to these OTUs were detected. Figure 7a shows the change in the proportion of respective OTUs from fecal samples obtained just before sacrifice. Depending on the conditions, the composition of the fecal microbiota differed. Due to differences in the original composition, a specific pattern of change was not observed. However, when the ratio of fecal microbiota obtained before and after the intervention was calculated, some OTUs showed changes according to experimental groups (Figure 7b). In particular, the proportion of OTU657, which was presumed to correspond to Lactobacillales in the WGtreated group, was increased dose-dependently compared to the DSS-treated group. The GR group also showed an increase in the proportion of OTU657. Furthermore, the proportion of OTU369, which was presumed to correspond to Clostridium cluster IV, was reduced by DSS treatment. Both WG and GR treatments showed a slight increase in the proportion of OTU 369. 


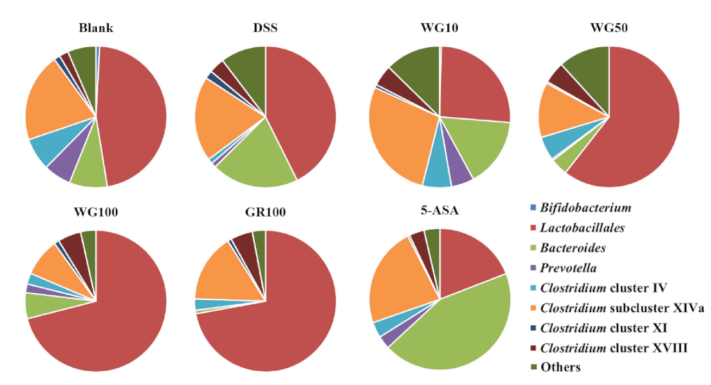

(a)
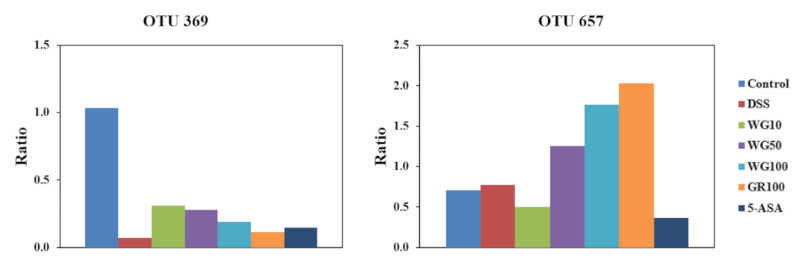

(b)

Figure 7. Stool sample microbiota from mice with DSS-induced ulcerative colitis, supplemented with or without WG and GR. (a) The relative abundance of bacteria in the stool was determined using terminal restriction fragment length polymorphism (T-RFLP) analysis. Data are shown as a percentage of the total identified sequences per group. (b) Effect of WG and GR on the ratio of operational taxonomic units (OTUs) before and after intervention with DSS, WG, GR, and 5-ASA.

\section{Discussion}

WG was developed to solve the problems of cultivation and the depletion of natural resources. To extend the utilization of the newly developed Glycyrrhiza variety, WG, several biological activities have been elucidated. In this study, the anti-inflammatory effect of WG on DSS-induced ulcerative colitis was studied.

UC is a chronic and complex autoimmune inflammatory disorder, also called chronic IBD, and is characterized by an uncontrolled inflammatory condition of the intestinal mucosa [27-30]. Symptoms of UC can include abdominal pain, bloody diarrhea, and weight loss [31]. In particular, the continuous progression of UC increases the risk of colorectal cancer [32]. Using 5-ASA, also known as mesalazine, yields immunosuppressive effects such as prostaglandin restriction and pro-inflammatory cytokine inhibition. The development of complementary treatments is needed for the effective mitigation of UC symptoms for safe long-term use due to the side effects of sulfasalazine [33,34]. Traditionally, GR is used for the treatment of allergies, bowel disease, and coughs [35]. GR possesses various bioactive constituents, including flavonoids, coumarins, and saponins [36]. Thus, we hypothesized that WG could also have a beneficial effect on UC. As shown in Figure 2, the DSS control group showed significantly increased body weight loss and decreased colon length in comparison with the control group. Previous studies have shown that the length change of the colon and the severity of ulcerative colitis are correlated [37]. In this study, we found that WG and GR effectively inhibited DSS-induced colon length, DAI, and weight loss-symptoms and pathological changes of colitis in our DSS model (Figure $3 \mathrm{a}, \mathrm{b}$ ). Macrophages and neutrophils penetrate the colon during $\mathrm{UC}$ and secrete large amounts of inflammatory cytokines, such as IL-6, IL-1 $\beta$, and TNF- $\alpha$ [38,39]. In IBD, immune cells such as macrophages and $\mathrm{T}$ lymphocytes secrete inflammatory cytokines [21,40]. These activated cells regulate the balance between increased TNF- $\alpha$ and IL- 6 pro-inflammatory cytokine expression in the rectal mucosa of UC patients [41]. In this study, WG and GR suppressed the DSS-induced increase in TNF- $\alpha$ and IL-6 levels in mouse serum (Figure 4). However, even with significant inhibition, the rates were variable by cytokine. In a previous study, GR extract inhibited IL-6, IL- $1 \beta$, and TNF- $\alpha$ in a different manner. IL-6 level was strongly suppressed by a treatment of $100 \mu \mathrm{g} / \mathrm{mL}$ in RAW 264.7 cells [42]. However, the TNF- $\alpha$ 
level showed less inhibition at same dose. This variability could mean that WG and GR selectively affect the production of cytokines based on dosing.

It is likely that the anti-inflammatory activities of WG and GR may be caused by glycyrrhetic acid, a metabolite of glycyrrhizin [43], as glycyrrhetic acid showed antiinflammatory activity via the inhibition of nuclear factor $\kappa \mathrm{B}(\mathrm{NF}-\mathrm{\kappa B})$ activation. As this mechanism has previously been reported, NF- $\kappa B$ was not analyzed in this study. However, NF- $k B^{\prime}$ s involvement in the anti-inflammatory activities of WG and GR is presumed, as glycyrrhizin was detected in relatively high quantities in both WG and GR extracts.

In the inflammatory response, cyclooxygenase (COX)-1 protein activity rarely changes; however, COX-2 production increases rapidly, leading to increased production of prostaglandin(PG)s [44], with especially high levels of $\mathrm{PGE}_{2}$ in the bowel of IBD patients [22]. $\mathrm{PGE}_{2}$ plays a key role as a mediator in UC. In the current study, WG and GR inhibited the DSS-induced activation of $\mathrm{PGE}_{2}$ production (Figure 5). As previously reported, the histopathological features of UC, such as ulceration, mucous membrane erosion, and loss of intestinal crypts, can be exhibited in DSS-induced colitis models. In the present study, WG $100 \mathrm{mg} / \mathrm{kg}$ and GR $100 \mathrm{mg} / \mathrm{kg}$ significantly reduced inflammatory cell infiltration versus the DSS-induced colitis model (Figure 6). Furthermore, bowel wall thickness associated with inflammation was suppressed by WG treatment (Figure 5a,c).

Because of the original composition of the fecal microbiota between samples, which might be one reason for the variation, significant differences were not observed. However, the proportion of Lactobacillales increased following the administration of WG and GR in the colitis model. In a previous report, the bacterial population of Lactobacillus decreased upon the administration of DSS to induce colitis [45]. GR, including WG consumption, might act as a prebiotic to improve DSS-induced colitis through the modulation of probiotics such as Lactobacillus. Licorice is categorized as a potential prebiotic and one of the major compounds, liquiritin, showed selective antibacterial activity to reduce pathogenic bacteria and maintain probiotics such as Bifidobacterium and Lactobacillus [46,47]. Further studies on the relationship between the improvement of DSS-induced colitis and changes in the composition of the fecal microbiota are needed to confirm the effect of WG.

In summary, the anti-inflammatory activities of WG and GR were equally effective as the positive control 5-ASA. WG inhibited inflammation by regulating inflammatory mediators such as IL-6, TNF- $\alpha$, and $\mathrm{PGE}_{2}$. WG may be a useful therapeutic agent to treat inflammatory diseases and utilized as an equivalent source of Glycyrrhizae radix for medicine.

\section{Materials and Methods}

\subsection{Reagents}

The Korea Rural Development Administration provided WG. GR was purchased from pharmaceutical company Humanherb (Kyuong San, Korea) and identified as G. uralensis by Dr. Jeonghoon Lee (RDA, Korea). Liquiritigenin was purchased from Interpharm (Ilsan, Gyeonggi-do, Korea). Glycyrrhizic acid was purchased from TCI (Tokyo, Japan).

Protein lysis buffer was obtained from iNtRON Biotech (Seongnam, Korea). DSS and 5-ASA were obtained from MP Bio and Sigma-Aldrich, respectively. Standard anti-mouse TNF- $\alpha$, IL-6, purified biotin, and TMB (3,3',5,5'-Tetramethylbenzidine) substrate reagent were obtained from BD Bioscience.

\subsection{Preparation of $W G$ and $G R$}

WG and GR (sliced) extracts were prepared by reflux decoction with ethanol (70\%) for $2 \mathrm{~h}$. The solvent was filtered and evaporated by a rotary evaporator. The yields of dried extract were $8.8 \%$ for WG and 3.0\% for GR. The extracts were diluted in purified water. The solutions were filtered by a $0.45 \mu \mathrm{m}$ syringe filter (HYUNDAI Micro). 


\subsection{LC/MS Analysis}

Methods for injection sample preparation and LC/MS analysis were modified from that of Korean Pharmacopoeia [48]. HPLC was carried out on a Waters 2695 system (Waters, USA) with a Waters 996 photodiode array (PDA) and a Waters micro mass ZQ mass spectrometer. The analysis of glycyrrhizic acid was performed under the following conditions: column, TSK-gel ODS-80Ts (Tosoh Co., Japan, $4.6 \mathrm{~mm} \times 150 \mathrm{~mm}$ ); mobile phase, $6.67 \%$ acetic acid (solvent system $\mathrm{A}$ ) and $\mathrm{CH}_{3} \mathrm{CN}$ (solvent system $\mathrm{B}$ ) in isocratic mode (solvent B $40 \%$ for $20 \mathrm{~min}$ ); flow rate: $1 \mathrm{~mL} / \mathrm{min}$; temperature: $30^{\circ} \mathrm{C}$; detection: $254 \mathrm{~nm}$. The analysis of liquiritigenin was performed under the following conditions: mobile phase, $1 \%$ acetic acid (solvent system $\mathrm{A}$ ) and $\mathrm{CH}_{3} \mathrm{CN}$ (solvent system $\mathrm{B}$ ) in isocratic mode (solvent B $25 \%$ for $20 \mathrm{~min}$ ); detection at $276 \mathrm{~nm}$. The mass spectrometer was operated in the negative ion mode. The total ion chromatogram was obtained using the following parameters: highpurity nitrogen was used as dry gas at a flow rate of $600 \mathrm{~L} / \mathrm{h}$, gas temperature of $400^{\circ} \mathrm{C}$, extractor voltage of $2 \mathrm{~V}$, cone voltage of $40 \mathrm{~V}$, and capillary voltage of $3 \mathrm{kV}$.

\subsection{Animals}

Male BALB/c, 7-week-old mice (19-21 g, $n=42)$ were purchased from SAMTACO (Osan, Korea). The mice were acclimatized in a specific pathogen free (SPF) environment for a week. The conditions were $22 \pm 2{ }^{\circ} \mathrm{C}$ and a 12-h light/dark cycle.

\subsection{DSS-Induced Ulcerative Colitis}

UC was induced by administering drinking water containing 3\% (w/v) DSS for 12 days. Forty-two mice were weighed and divided into seven groups with six mice per group: (i) normal group; (ii) DSS-induced colitis group; (iii) DSS-induced colitis + WG $10 \mathrm{mg} / \mathrm{kg}$ group; (iv) DSS-induced colitis + WG $50 \mathrm{mg} / \mathrm{kg}$ group; (v) DSS-induced colitis + WG $100 \mathrm{mg} / \mathrm{kg}$ group; (vi) DSS-induced colitis + GR $100 \mathrm{mg} / \mathrm{kg}$ group; and (vii) DSSinduced colitis + 5-ASA group. Body weight, stool consistency, and gross bleeding of the animals were checked daily.

\subsection{Disease Activity Index (DAI)}

A DAI score was determined by the method described by Murthy et al. (Table 1) [49].

Table 1. Disease activity index.

\begin{tabular}{cccc}
\hline Score & Stool Consistency & Occult/Gross & Weight Loss (\%) \\
\hline 0 & Normal & Normal & $(-)$ \\
1 & & & $1-5$ \\
2 & Loose & Guiac $(+)$ & $5-10$ \\
3 & & & $10-15$ \\
4 & Diarrhea & Gross bleeding & $>15$ \\
\hline
\end{tabular}

$\overline{\mathrm{DAI}}=($ Combined score of stool consistency, rectal bleeding, and body weight loss $) / 3$.

\subsection{ELISA Measurement}

At the end of the experiment, serum samples were prepared and subjected to ELISAs to determine the serum levels of IL- 6 and TNF- $\alpha$ using ELISA kits from BD Pharmingen. Absorbance at $450 \mathrm{~nm}$ was measured using a microplate reader.

\subsection{Prostaglandin $E_{2}\left(P_{G} E_{2}\right)$ Assay}

Whole mouse colons were washed with cold PBS and immediately stored at $-80{ }^{\circ} \mathrm{C}$ until use. Colon tissues were homogenized in $1 \mathrm{~mL}$ of ice-cold lysis buffer (iNtRon) using a homogenizer. Tissue homogenates were vortexed and centrifuged at $4{ }^{\circ} \mathrm{C}$ for $10 \mathrm{~min}$ at $12,000 \mathrm{rpm}$. The supernatant $(5 \mu \mathrm{L})$ from each sample was used to determine the protein concentration using a DC Protein Assay Kit (Bio-Rad, Hercules, USA). PGE $_{2}$ levels were determined using a $\mathrm{PGE}_{2}$ Monoclonal Enzyme Immunoassay Kit (Enzo Bioscience). All samples were diluted with assay buffer at 1:100. Experiments were run in triplicate and 
a standard curve was created concurrently. Absorbance at $405 \mathrm{~nm}$ was measured using a microplate reader.

\subsection{Histological Processing}

Specimens of the colon were fixed with $10 \%$ buffered formalin and embedded in paraffin. The sections $(4 \mu \mathrm{m})$ were stained with H\&E and examined by light microscopy for the presence of lesions. The severity of colitis was evaluated in sections stained with hematoxylin and eosin by two independent observers who were blinded to the experimental conditions according to modified criteria [50] summarized in Table 2.

Table 2. Criteria for assessment of microscopic rectal damage.

\begin{tabular}{cc}
\hline Score & Remarks \\
\hline 1 & Normal colonic mucosa \\
2 & Loss of one-third of the crypts \\
3 & Loss of two-thirds of the crypts \\
4 & Lamina propria covered with single layer of epithelial cells with mild \\
5 & inflammatory cell infiltration \\
$\begin{array}{l}\text { Mucosal damage was scored as } 0-4 \text { based on the loss of crypts (mucosa) and infiltration of inflammatory cells } \\
\text { (maximum score = 5). }\end{array}$
\end{tabular}

\subsection{DNA Isolation from Fecal Samples}

Terminal restriction fragment length polymorphism (T-RFLP) was analyzed as described previously $[25,26]$. Total DNA was extracted using a HiGene Genomic DNA Prep Kit as described in the protocol (BioFACT, Yuseong-Gu, South Korea) after homogenizing the feces with bead-beating $2 \times 25 \mathrm{~s}$ using Precellys 24 (Bertin, France) at $6500 \mathrm{rpm}$. The DNA preparation was purified using a high-purity PCR template preparation kit (Roche).

\subsection{PCR Amplification}

The 16S rRNA genes were amplified using primers $5^{\prime}$ FAM-labeled 516f (5'-TGCCA GCAGCCGCGGTA-3') and 1510r (5'-GGTTACCTTGTTACGACTT-3'). The PCR mixture, in a total volume of $50 \mu \mathrm{L}$, contained $20 \mathrm{pmol}$ of each primer (FAM-516f and 1510r), $5 \mu \mathrm{L}$ of $10 \times$ PCR buffer, $10 \mathrm{mM}$ of each dNTP (nucleoside triphosphate), $2.5 \mathrm{U}$ of A-Star Lamp Taq DNA Polymerase (BioFACT, Yuseong-Gu, South Korea), and $2 \mu \mathrm{L}$ of template DNA. The PCR was conducted in a thermal cycler (Applied Biosystems) using the following program: $95^{\circ} \mathrm{C}$ for $3 \mathrm{~min}, 30$ cycles of $95^{\circ} \mathrm{C}$ for $30 \mathrm{~s}, 55^{\circ} \mathrm{C}$ for $30 \mathrm{~s}, 72^{\circ} \mathrm{C}$ for $30 \mathrm{~s}$, and finally $70^{\circ} \mathrm{C}$ for $10 \mathrm{~min}$. After amplification, the PCR products were confirmed by agarose gel electrophoresis. The PCR products were purified using the HiGene Gel \& PCR Purification System (BioFACT, Yuseong-Gu, South Korea).

\subsection{T-RFLP Analysis}

The PCR products were separately digested with $10 \mathrm{U}$ of the restriction endonuclease $\mathrm{Bsl} \mathrm{I}$ (Thermo Scientific) in a total volume of $30 \mu \mathrm{L}$ for $2 \mathrm{~h}$ at $55^{\circ} \mathrm{C}$. Analysis was conducted on a 3130xl Genetic Analyzer (Applied Biosystems), and a DNA size marker MM1000-ROX (BioVentures) was included in all samples. Fragment sizes and peak area were analyzed with Genemapper ${ }^{\circledR}$ (Applied Biosystems).

\subsection{Assignment of Terminal Restriction Fragments Obtained}

An operational taxonomic unit (OTU) was adopted to distribute the T-RFs. Twentynine OTUs were obtained based on the method of Nagashima et al. [51] and the database for T-RFLP analysis of the human colonic microbiota [52]. 


\subsection{Statistical Analysis}

The results are presented as the mean \pm SEM of at least three independent experiments. The results were analyzed using SPSS Statistics 20 (IBM Corp, Armonk, NY). The Tukey test was used to determine statistically significant differences. $P<0.05$ was considered significant.

\section{Conclusions}

In conclusion, WG and GR extracts show clinical effectiveness for DSS-induced colitis by reducing levels of inflammatory mediators and suppression of $\mathrm{PGE}_{2}$ production. Furthermore, WG shows equivalent efficacy as conventional GR, and therefore WG can be added to the compendia as a potential treatment for DSS-induced colitis.

Author Contributions: Conceptualization, J.-S.J. and S.-H.K.; formal analysis, Y.-J.S. and D.-K.K.; investigation, Y.-D.J.; resources, J.L. and J.-K.C.; data curation, S.-H.K. and J.-H.P.; writing—original draft preparation, J.-S.J. and S.-H.K.; writing-review and editing, J.-S.J., C.K. and S.-H.K.; visualization, S.-H.K. and J.-R.S.; supervision, J.-S.J. and J.-H.L.; project administration, J.-S.J., W.K. and H.-J.A. All authors have read and agreed to the published version of the manuscript.

Funding: This work was carried out with the support of "Cooperative Research Program for Agriculture Science and Technology Development (Project No. PJ01424602)" Rural Development Administration, Republic of Korea.

Institutional Review Board Statement: The study was conducted according to the guidelines of the Declaration of Helsinki and approved by the Institutional Review Board of Jeonbuk National University (CBNU2020-0105).

Informed Consent Statement: Not applicable.

Data Availability Statement: The data are available from the corresponding author upon request.

Conflicts of Interest: The authors declare that they have no conflict of interest.

\section{References}

1. Pastorino, G.; Cornara, L.; Soares, S.; Rodrigues, F.; Oliveira, M. Glycyrrhiza (Glycyrrhiza glabra): A phytochemical and pharma-cological review. Phytother. Res. 2018, 32, 2323-2339. [CrossRef]

2. Committee of Jiangsu New Medical College. Encyclopedia of Traditional Chinese Medicine; Science and Technology Press: Shanghai, China, 1995; p. 657.

3. Hosseinzadeh, H.; Nassiri-Asl, M. Pharmacological Effects of Glycyrrhiza spp. and Its Bioactive Constituents: Update and Review. Phytother. Res. 2015, 29, 1868-1886. [CrossRef]

4. Park, C.G.; Lee, J.H.; Kim, O.T.; Park, C.B.; Kim, G.S.; Ahn, Y.S.; Cha, S.W.; Lee, S.H.; Kim, M.S.; Heo, C.S.; et al. A new Glycyrrhiza variety "Wongam" through interspecific cross between Glycyrrhiza glabra and Glycyrrhiza uralensis. Korean J. Med. Crop Sci. 2014, 22, 169-170.

5. Lee, S.E.; Lee, J.H.; Park, C.G.; Kim, H.D.; Lee, Y.; Seo, K.H.; Jeong, H.S.; Jang, J.K.; Kim, D.H. Evaluation of the In vitro Activity of Glycyrrhiza Cultivar Roots. Korean J. Med. Crop Sci. 2019, 27, 115-125. [CrossRef]

6. Rubin, D.C.; Shaker, A.; Levin, M.S. Chronic intestinal inflammation: Inflammatory bowel disease and colitis-associated colon cancer. Front. Immunol. 2012, 3, 107. [CrossRef]

7. Kappelman, M.D.; Rifas-Shiman, S.L.; Kleinman, K.; Ollendorf, D.; Bousvaros, A.; Grand, R.J.; Finkelstein, J.A. The Prevalence and Geographic Distribution of Crohn's Disease and Ulcerative Colitis in the United States. Clin. Gastroenterol. Hepatol. 2007, 5, 1424-1429. [CrossRef] [PubMed]

8. Lennard-Jones, J.E. Classification of Inflammatory Bowel Disease. Scand. J. Gastroenterol. 1989, 24, 2-6. [CrossRef]

9. Sartor, R.B. Current concepts of the etiology and pathogenesis of ulcerative colitis and Crohn's disease. Gastroenterol. Clin. N. Am. $1995,24,475-507$.

10. Head, K.A.; Jurenka, J.S. Inflammatory bowel disease part I: Ulcerative colitis—Pathophysiology and conventional and alternative treatment options. Altern. Med. Rev. 2003, 8, 247-283.

11. Cosnes, J.; Gower-Rousseau, C.; Seksik, P.; Cortot, A. Epidemiology and Natural History of Inflammatory Bowel Diseases. Gastroenterology 2011, 140, 1785-1794.e4. [CrossRef] [PubMed]

12. Cipolla, G.; Crema, F.; Sacco, S.; Moro, E.; De Ponti, F.; Frigo, G. Nonsteroidal anti-inflammatory drugs and inflammatory bowel disease: Current perspectives. Pharmacol. Res. 2002, 46, 1-6. [CrossRef]

13. Soufli, I.; Toumi, R.; Rafa, H.; Touil-Boukoffa, C. Overview of cytokines and nitric oxide involvement in immuno-pathogenesis of inflammatory bowel diseases. World J. Gastrointest. Pharmacol. Ther. 2016, 7, 353-360. [CrossRef] 
14. Katsanos, K.H.; Papadakis, K.A. Inflammatory Bowel Disease: Updates on Molecular Targets for Biologics. Gut Liver 2017, 11, 455-463. [CrossRef]

15. Chassaing, B.; Aitken, J.D.; Malleshappa, M.; Vijay-Kumar, M. Dextran Sulfate Sodium (DSS)-Induced Colitis in Mice. Curr. Protoc. Immunol. 2014, 104, 15.25.1-15.25.14. [CrossRef]

16. Wirtz, S.; Neufert, C.; Weigmann, B.; Neurath, M.F. Chemically induced mouse models of intestinal inflammation. Nat. Protoc. 2007, 2, 541-546. [CrossRef]

17. Choi, C.H.; Moon, W.; Kim, Y.S.; Kim, E.S.; Lee, B.-I.; Jung, Y.; Yoon, Y.S.; Lee, H.; Park, D.I.; Han, D.S.; et al. Second Korean guidelines for the management of ulcerative colitis. Intest. Res. 2017, 15, 7-37. [CrossRef]

18. Lakatos, L. Immunology of inflammatory bowel diseases. Acta Physiol. Hung. 2000, 87, 355-372. [PubMed]

19. Inoue, S.; Matsumoto, T.; Iida, M.; Mizuno, M.; Kuroki, F.; Hoshika, K.; Shimizu, M. Characterization of cytokine expression in the rectal mucosa of ulcerative colitis: Correlation with disease activity. Am. J. Gastroenterol. 1999, 94, 2441-2446. [CrossRef] [PubMed]

20. Hendrickson, B.A.; Gokhale, R.; Cho, J.H. Clinical Aspects and Pathophysiology of Inflammatory Bowel Disease. Clin. Microbiol. Rev. 2002, 15, 79-94. [CrossRef]

21. Naito, Y.; Takagi, T.; Uchiyama, K.; Kuroda, M.; Kokura, S.; Ichikawa, H.; Yanagisawa, R.; Inoue, K.-I.; Takano, H.; Yoshikawa, T.; et al. Reduced intestinal inflammation induced by dextran sodium sulfate in interleu-kin-6-deficient mice. Int. J. Mol. Med. 2004, 14, 191-196.

22. Wiercin'ska-Drapało, A.; Flisiak, R.; Prokopowicz, D. Effects of ulcerative colitis activity on plasma and mucosal prostaglandin E2 concentration. Prostaglandins Other Lipid Mediat. 1999, 58, 159-165. [CrossRef]

23. Kim, D.S.; Ko, J.H.; Jeon, Y.D.; Han, Y.H.; Kim, H.J.; Poudel, A.; Jung, H.-J.; Ku, S.-K.; Kim, S.-J.; Hong, S.H.; et al. Ixeris dentate NAKAI reduces clinical score and HIF-1 expression in experimental colitis in mice. Evid. Based Complement. Altern. Med. 2013, 2013, 671281. [CrossRef] [PubMed]

24. Krych, L.; Kot, W.; Bendtsen, K.M.; Hansen, A.K.; Vogensen, F.K.; Nielsen, D.S. Have you tried spermine? A rapid and cost-effective method to eliminate dextran sodium sulfate inhibition of PCR and RT-PCR. J. Microbiol. Methods 2018, 144, 1-7. [CrossRef] [PubMed]

25. Jin, J.S.; Touyama, M.; Hisada, T.; Benno, Y. Efects of green tea consumption on human fecal microbiota with special reference to Bifdobacterium species. Microbiol. Immunol. 2012, 56, 729-739. [CrossRef] [PubMed]

26. Protective effect of agaro-oligosaccharides on gut dysbiosis and colon tumorigenesis in high-fat diet-fed mice. Am. J. Physiol. Gastrointest. Liver Physiol. 2016, 310, G367-G375. [CrossRef]

27. Dharmani, P.; Chadee, K. Biologic therapies against inflammatory bowel disease: A dysregulated immune system and the cross talk with gastrointestinal mucosa hold the key. Curr. Mol. Pharmacol. 2008, 1, 195-212. [CrossRef]

28. Strober, W.; Fuss, I.; Mannon, P. The fundamental basis of inflammatory bowel disease. J. Clin. Investig. 2007, 117, 514-521. [CrossRef]

29. Sartor, R.B. Microbial Influences in Inflammatory Bowel Diseases. Gastroenterology 2008, 134, 577-594. [CrossRef]

30. Podolsky, D.K. Inflammatory bowel disease. N. Engl. J. Med. 2002, 347, 417-429. [CrossRef]

31. Rufo, P.A.; Bousvaros, A. Current therapy of inflammatory bowel disease in children. Pediatr. Drugs 2006, 8, 279-302. [CrossRef] [PubMed]

32. Jin, B.R.; Chung, K.S.; Cheon, S.Y.; Lee, M.; Hwang, S.; Noh Hwang, S.; Rhee, K.J.; An, H.J. Rosmarinic acid suppresses colonic in-flammation in dextran sulphate sodium (DSS)-induced mice via dual inhibition of NF- $\mathrm{KB}$ and STAT3 activation. Sci. Rep. 2017, 7,45252 .

33. Rogler, G. Gastrointestinal and liver adverse effects of drugs used for treating IBD. Best Pract. Res. Clin. Gastroenterol. 2010, 24, 157-165. [CrossRef]

34. Pandurangan, A.K.; Ismail, S.; Saadatdoust, Z.; Esa, N.M. Allicin Alleviates Dextran Sodium Sulfate- (DSS-) Induced Ulcerative Colitis in BALB/c Mice. Oxid. Med. Cell. Longev. 2015, 2015, 1-13. [CrossRef]

35. Zu, Y.; Wang, S.; Luo, M.; Fu, Y.; Efferth, T. Glycyrrhizic acid nanoparticles inhibit LPS-induced inflammatory mediators in 264.7 mouse macrophages compared with unprocessed glycyrrhizic acid. Int. J. Nanomed. 2013, 8, 1377-1383. [CrossRef] [PubMed]

36. Chang, Y.L.; Chen, C.L.; Kuo, C.L.; Chen, B.C.; You, J.S. Glycyrrhetinic acid inhibits ICAM-1 expression via blocking JNK and NF-кB pathways in TNF- $\alpha$-activated endothelial cells. Acta Pharmacol. Sin. 2010, 31, 546-553. [CrossRef]

37. Kang, S.H.; Jeon, Y.D.; Moon, K.H.; Lee, J.H.; Kim, D.G.; Kim, W.; Myung, H.; Kim, J.-S.; Kim, H.-J.; Jin, J.S.; et al. Aronia Berry Extract Ameliorates the Severity of Dextran Sodium Sulfate-Induced Ul-cerative Colitis in Mice. J. Med. Food 2017, 20, 667-675. [CrossRef] [PubMed]

38. Guimbaud, R.; Bertrand, V.; Chauvelot-Moachon, L.; Quartier, G.; Vidon, N.; Giroud, J.-P.; Couturier, D.; Chaussade, S. Network of inflammatory cytokines and correlation with disease activity in ulcerative colitis. Am. J. Gastroenterol. 1998, 93, $2397-2404$. [CrossRef]

39. Hegazy, S.K.; El-Bedewy, M.M. Effect of probiotics on pro-inflammatory cytokines and NF-кB activation in ulcerative colitis. World J. Gastroenterol. 2010, 16, 41-45. [CrossRef]

40. Myers, K.J.; Murthy, S.; Flanigan, A.; Witchell, D.R.; Butler, M.; Murray, S.; Siwkowski, A.; Goodfellow, D.; Madsen, K.; Baker, B. Antisense Oligonucleotide Blockade of Tumor Necrosis Factor- $\alpha$ in Two Murine Models of Colitis. J. Pharmacol. Exp. Ther. 2003, 304, 411-424. [CrossRef] 
41. Peng, J.C.; Shen, J.; Ran, Z.-H. Novel agents in the future: Therapy beyond anti-TNF agents in inflammatory bowel disease. J. Dig. Dis. 2014, 15, 585-590. [CrossRef]

42. Jeon, Y.D.; Kang, S.H.; Bang, K.S.; Chang, Y.N.; Lee, J.H.; Jin, J.S. Glycyrrhetic Acid Ameliorates Dextran Sulfate Sodium-Induced Ul-cerative Colitis in Vivo. Molecules 2016, 21, 523. [CrossRef]

43. Morita, I. Distinct functions of COX-1 and COX-2. Prostaglandins Other Lipid Mediat. 2002, 68, 165-175. [CrossRef]

44. Lauritsen, K.; Laursen, L.S.; Kjeldsen, J.; Bukhave, K.; Hansen, T.K.; Rask-Madsen, J. Effects of mesalazine on the formation of lipoxygenase and cyclooxygenase products. Single Mol. Single Cell Seq. 1995, 371, 1301-1306.

45. Håkansson, Å.; Tormo-Badia, N.; Baridi, A.; Xu, J.; Molin, G.; Hagslätt, M.L.; Karlsson, C.; Jeppsson, B.; Cilio, C.M.; Ahrné, S. Immunological alteration and changes of gut microbiota after dextran sulfate sodium (DSS) administration in mice. Clin. Exp. Med. 2015, 15, 107-120. [CrossRef]

46. Peterson, C.T.; Sharma, V.; Uchitel, S.; Denniston, K.; Chopra, D.; Mills, P.J.; Peterson, S.N. Prebiotic Potential of Herbal Medicines Used in Digestive Health and Disease. J. Altern. Complement. Med. 2018, 24, 656-665. [CrossRef] [PubMed]

47. Zhang, W.; Jiang, S.; Qian, D.; Shang, E.; Duan, J. Effect of liquiritin on human intestinal bacteria growth: Metabolism and modulation. Biomed. Chromatogr. 2014, 28, 1271-1277. [CrossRef]

48. Ministry of Food and Drug Safety. Korean Pharmaceutical Articles Part 2 (No. 2019-102); MFDS: Cheongju-si, Korea, $2019 ;$ pp. 5-6.

49. Murthy, S.N.S.; Cooper, H.S.; Shim, H.; Shah, R.S.; Ibrahim, S.A.; Sedergran, D.J. Treatment of dextran sulfate sodium-induced murine colitis by intracolonic cyclosporin. Dig. Dis. Sci. 1993, 38, 1722-1734. [CrossRef]

50. Hamamoto, N.; Maemura, K.; Hirata, I.; Murano, M.; Sasaki, S.; Katsu, K. Inhibition of dextran sulphate sodium (DSS)-induced colitis in mice by intracolonically administered antibodies against adhesion molecules (endothelial leucocyte adhesion mole-cule-1 (ELAM-1) or intercellular adhesion molecule-1 (icam-1)). Clin. Exp. Immunol. 1999, 117, 462-468. [CrossRef]

51. Nagashima, K.; Mochizuki, J.; Hisada, T.; Suzuki, S.; Shimomura, K. Phylogenetic Analysis of 16S Ribosomal RNA Gene Sequences from Human Fecal Microbiota and Improved Utility of Terminal Restriction Fragment Length Polymorphism Profiling. Biosci. Microflora 2006, 25, 99-107. [CrossRef]

52. Shyu, C.; Soule, T.; Bent, S.J.; Foster, J.A.; Forney, L.J. MiCA: A Web-Based Tool for the Analysis of Microbial Communities Based on Terminal-Restriction Fragment Length Polymorphisms of 16S and 18S rRNA Genes. Microb. Ecol. 2007, 53, 562-570. [CrossRef] [PubMed] 